\title{
Approach to Calculate the Degree of Similarity between Generalized Trapezoidal Fuzzy Numbers
}

\author{
Aditya Kumar Anand \\ Research Scholar, Department of Mathematics \\ L. N. M. U, Darbhanga \\ Bihar, India
}

\begin{abstract}
This paper presents a new statistical approach to measure the degree of similarity between the generalized trapezoidal fuzzy numbers. Since the evolution of Fuzzy sets and logic, many researchers, mathematicians are working on the Fuzzy systems to find unhidden concepts related to it. It has been used in almost every sphere, whether it is a scientific research analysis or the pattern recognition, decision-making or the image processing. These fields and many more, widely use the similarity measure of fuzzy numbers. In this work, a simple method has been proposed to calculate the degree of similarity between the fuzzy numbers. It is one of the simplest method to find the degree of similarity between the fuzzy numbers. Complexity is reduced by utilizing the least number of parameters to calculate the degree of similarity. A comparative study between the proposed model and the existing similarity model shows that the proposed method is more accurate than the existing models, which are used to find the degree of similarity between the fuzzy numbers.
\end{abstract}

\section{Keywords}

Degree of Similarity, Similarity Measures, Fuzzy Numbers, Generalized Trapezoidal Fuzzy Numbers

\section{INTRODUCTION}

"Fuzzy" evolved in early 1970's, have dictionary meaning as shapes that do not have clear edges. This term is generally used for shapes or structures which is difficult to perceive; indistinct or vague. In mathematics, fuzzy relates to a form of set theory and logic which predicates, degrees of applicability, unlike Boolean logic which means simply being true or false. It describes the uncertainty in the universe in more clear way than that described by Boolean systems. It has been accounted in the researches that fuzzy numbers describes the physical world more realistically than "ordinary" numbers. This is one of main reason that the generalized fuzzy numbers have been widely used in many areas including statistics, computer science, and engineering.

When the fuzzy numbers come into picture than the degree of similarity between the fuzzy numbers become the most important term. Over the last decade many new methods has been proposed, to calculate the degree of similarity between the generalized trapezoidal fuzzy numbers. But, it remains a subject on which research are still going on to find the most accurate, optimized and error free method to obtain degree of similarity between the generalized trapezoidal fuzzy numbers. Degree of similarity has been applied in many scientific application as documented in [1-7]. With different method there remain some difficulty to calculate the degree of similarity between the generalized trapezoidal fuzzy numbers. However, since fuzzy numbers are represented by possibility distributions, they can overlap with each other and, as a result, it is not easy to develop an effective similarity measure. In particular, existing similarity measures sometime not able to obtain the degree of similarity between the generalized trapezoidal fuzzy numbers and the existing approach are very complex.

In this paper, a novel similarity measure of generalized trapezoidal fuzzy numbers is proposed. The proposed similarity measure of generalized trapezoidal fuzzy numbers has a simple definition and is easier to understand. The proposed approach has been compared with the existing models and obtained result shows that the proposed method is more accurate and simple than the existing models.

This paper has been organized in five sections. Section 1 details the introduction to the research work. Section 2 presents the mathematical overview of the subject. Section 3 brief the concepts of the existing similarity measure and the proposed Similarity Measure. Further section 4, gives the comparative study performed. Section 5 presents the conclusion of the research work.

\section{FUZZY: MATHEMATICAL REVIEW 2.1 Fuzzy Sets}

A fuzzy set, $\tilde{A}$ in a universe of discourse $\mathrm{X}$ is defined as the following set of pairs $A=\left\{\left(x, \mu_{\tilde{A}}(x): x \in X\right)\right\}$. Here, $\mu_{\tilde{A}}$ : $X \rightarrow[0,1]$ is a mapping called as the membership value of $\mathrm{x} \epsilon$ $\mathrm{X}$ in a fuzzy set $\tilde{A}$.

\subsection{Alpha-Cut of Fuzzy Sets}

A $\alpha$ - level set of the fuzzy set $\tilde{A}$ of $\mathrm{X}$ is a crisp set A $\propto$ which contains all the elements of $\mathrm{X}$ that have membership values in A greater than or equal to $\alpha$ i.e., $A_{\propto}=\left\{\left(x: \mu_{\tilde{A}}(x) \geq \propto\right.\right.$ , $x \in X, \propto \in[0,1]$.

\subsection{Convex Fuzzy Sets}

A fuzzy set $\mathrm{A}=\left\{\left(\mathrm{x}, \mu_{\mathrm{A}}(\mathrm{x})\right)\right\} \subseteq \mathrm{X}$ is called fuzzy set if all $\mathrm{A}_{\propto}$ are convex sets i.e., for every element $\mathrm{x}_{1} \in \mathrm{A}_{\alpha}$ and $\mathrm{x}_{2} \in \mathrm{A}_{\alpha}$ and for every $\alpha \in[0,1] \lambda \mathrm{x}_{1}+(1-\lambda) \mathrm{x}_{2} \in \mathrm{A}_{\alpha} \forall \lambda \in[0,1]$.

If these conditions are not satisfied then the fuzzy set is called as the non-convex fuzzy set.

\subsection{Fuzzy Numbers}

A fuzzy number is the fuzzy subset of the real line for which the highest membership values are clustered around a given real number. It is expressed as a fuzzy set defining a fuzzy interval in the real number.

\subsection{Interval Number}

A real number $\Re$, interval on this is said to be a subset of $\Re$. If interval is denoted by $A=\left[a_{1}, a_{3}\right] a_{1}, a_{3} \in \Re, a_{1}<a_{3}$, this may 
be regarded as one sets. Interval is defined as having the membership function as shown in the Figure 1.

Membership function is defined as:

$$
\mu_{A}(\mathrm{x})=\left\{\begin{array}{lr}
0 & x<a_{1} \\
1 & a_{1} \leq x \leq a_{2} \\
0 & x>a_{3}
\end{array}\right.
$$

If $\mathrm{a}_{1}=\mathrm{a}_{3}$, this interval indicates a point. That is, $\left[\mathrm{a}_{1}, \mathrm{a}_{1}\right]=\mathrm{a}_{1}$

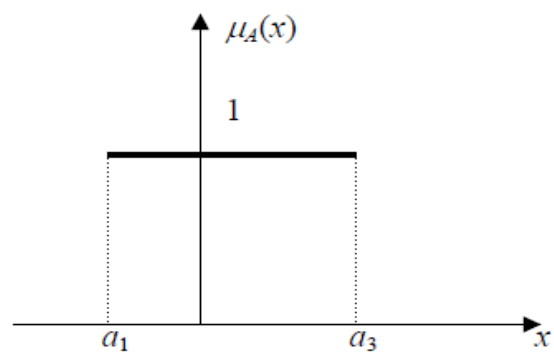

Figure 1 Interval $A=\left[a_{1}, a_{3}\right]$

\subsection{Trapezoidal Fuzzy Numbers}

A Trapezoidal fuzzy number $(\mathrm{TrFN})$ as presented in Figure 2.3. It may be denoted by $A$, as $\left(a_{1}, a_{2}, a_{3}, a_{4}\right)$ where the membership function is given as follows:

$$
\mu_{\tilde{A}}(x)= \begin{cases}0, & \mathrm{x}<\mathrm{a}_{1} \\ \left(\mathrm{x}-\mathrm{a}_{1}\right) /\left(\mathrm{a}_{2}-\mathrm{a}_{1}\right), & \mathrm{a}_{1} \leq \mathrm{x} \leq \mathrm{a}_{2} \\ 1, & \mathrm{a}_{2} \leq \mathrm{x} \leq \mathrm{a}_{3} \\ \left(\mathrm{a}_{4}-\mathrm{x}\right) /\left(\mathrm{a}_{4}-\mathrm{a}_{3}\right), & \mathrm{a}_{3} \leq \mathrm{x} \leq \mathrm{a}_{4} \\ 0, & \mathrm{x}>\mathrm{a}_{4} .\end{cases}
$$

or, it may be represented as :

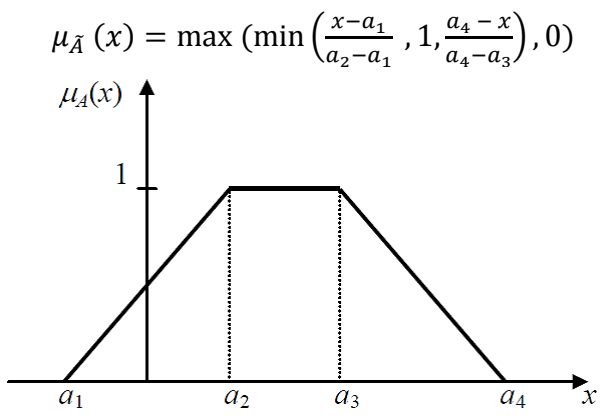

Figure 2 Trapezoidal fuzzy number $A=(\mathbf{a} 1, \mathbf{a} 2, \mathbf{a 3}, \mathbf{a 4})$

\subsection{Generalized Fuzzy Numbers}

A fuzzy subset $\tilde{A}$ of the real line is called a generalized fuzzy number if its membership function $\mu \tilde{A}$ has the following properties:

1. $\mu \tilde{A}$ is a continuous mapping from $-\infty$ to the closed interval $[0, \mathrm{~h}]$;

2. $\mu \tilde{A}(\mathrm{x})=0$ for $-\infty<\mathrm{x}<\mathrm{a}$;

3. $\mu \tilde{A}(\mathrm{x})=1 \tilde{\mathrm{A}}(\mathrm{x})$ is strictly increasing on $[\mathrm{a}, \mathrm{b}]$;

4. $\mu \tilde{A}(\mathrm{x})=\mathrm{h}$ for $\mathrm{b} \leq \mathrm{x} \leq \mathrm{c}$;
5. $\mu \tilde{A}(\mathrm{x})=\mathrm{r} \tilde{\mathrm{A}}(\mathrm{x})$ is strictly decreasing on $[\mathrm{c}, \mathrm{d}]$;

6. $\mu \tilde{A}(\mathrm{x})=0$ for all $\mathrm{d} \leq \mathrm{x}<\infty$;

where $0<\mathrm{h} \leq 1, \mathrm{a}, \mathrm{b}, \mathrm{c}, \mathrm{d}$ are real numbers, $1 \tilde{\mathrm{A}}$ and $\mathrm{r} \tilde{\mathrm{A}}$ are called the left membership function and the right membership function, respectively.

\subsection{Generalized Trapezoidal Fuzzy Number (GTrFN)}

A Generalized Fuzzy Number A is show in Figure 3. It may be represented as $\left(a_{1}, a_{2}, a_{3}, a_{4} ; w\right)$. A Generalized Trapezoidal Fuzzy Number has the following membership function:

$$
\mu_{A}(x)= \begin{cases}0, & x<a \\ h(x-a) /(b-a), & a \leq x<b \\ h, & b \leq x \leq c \\ h(a-c) /(d-c), & c<x \leq d \\ 0, & x>d .\end{cases}
$$

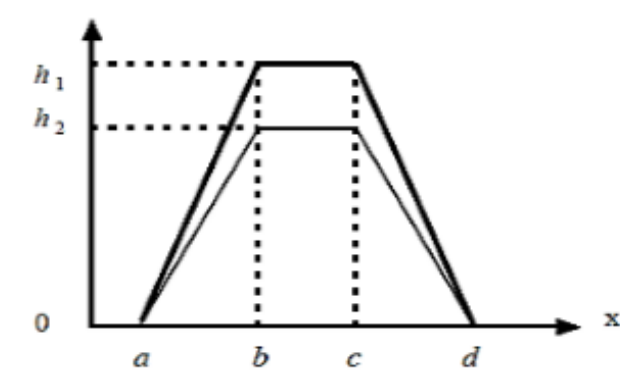

Figure 3 Two Generalized Trapezoidal Fuzzy Numbers

Figure 3 shows two different generalized trapezoidal fuzzy numbers $\tilde{\mathrm{A}}=\left(\mathrm{a}, \mathrm{b}, \mathrm{c}, \mathrm{d} ; \mathrm{h}_{1}\right)$ and $\mathrm{B}=\left(\mathrm{a}, \mathrm{b}, \mathrm{c}, \mathrm{d} ; \mathrm{h}_{2}\right)$. Here, $\mathrm{h}_{1}$ and $h_{2}$ represent the degrees of confidence.

\section{SIMILARITY MEASURES}

A similarity measure is a function $S: T F[0,1] X T F[0,1] \rightarrow$ $[0,1]$; indicating the degree of similarity between two fuzzy numbers. The closer this value is to 1 , the more similar the fuzzy numbers will be to each other.

\subsection{Similarity Measure Literature}

Let, there be two trapezoidal fuzzy numbers represented as A $=\left(a_{1}, a_{2}, a_{3}, a_{4}\right)$ and $B=\left(b_{1}, b_{2}, b_{3}, b_{4}\right)$. The first ideas about the similarity of normalized fuzzy numbers is obtained from the distance notion. If the distance between the fuzzy numbers $\mathrm{A}$ and $\mathrm{B}$ is obtained then the similarity function, $\mathrm{S}$ between them can be calculated by subtracting the distance value from 1 i.e., $S=1-d$.

In [8] and [9], Chen defined the degree of similarity $S_{C}(A, B)$ between two trapezoidal fuzzy numbers.

$$
S_{C}(A, B)=1-\frac{\sum_{i=1}^{4}\left|a_{i}-b_{i}\right|}{4}
$$

Where $S_{C}(A, B) \in[0,1]$. If $\mathrm{A}$ and $\mathrm{B}$ are two triangular fuzzy numbers, where $\mathrm{A}=\left(\mathrm{a} 1, \mathrm{a}_{2}, \mathrm{a}_{3}\right)$ and $\mathrm{B}=\left(\mathrm{b}_{1}, \mathrm{~b}_{2}, \mathrm{~b} 3\right)$, then the degree of similarity $S_{C}(A, B)$ between $\mathrm{A}$ and $\mathrm{B}$ can be calculated as follows: 


$$
S_{C}(A, B)=1-\frac{\sum_{i=1}^{3}\left|a_{i}-b_{i}\right|}{3}
$$

In [10], Hsieh et. al., proposed a similarity measure based on the idea of graded mean integration-representation distance, where the degree of similarity $S_{H C}(A, B)$ between two fuzzy numbers $\mathrm{A}$ and $\mathrm{B}$ was defined as follows:

$$
S_{H C}(A, B)=\frac{1}{1+d(A, B)}
$$

Where, $d(A)=|P(A)-P(B)| . \mathrm{P}(\mathrm{A})$ and $\mathrm{P}(\mathrm{B})$ are the graded mean integration representations of $\mathrm{A}$ and $\mathrm{B}$, respectively.

If $\mathrm{A}$ and $\mathrm{B}$ are two triangular fuzzy numbers, where $\mathrm{A}=(\mathrm{a} 1$, $\mathrm{a} 2$, a3) and $\mathrm{B}=(\mathrm{b} 1, \mathrm{~b} 2, \mathrm{~b} 3)$, then we have

$$
\begin{aligned}
& P(A)=\frac{a_{1}+4 a_{2}+a_{3}}{6} \\
& P(B)=\frac{b_{1}+4 b_{2}+b_{3}}{6}
\end{aligned}
$$

If $\mathrm{A}$ and $\mathrm{B}$ are trapezoidal fuzzy numbers, where $\mathrm{A}=(\mathrm{a} 1, \mathrm{a} 2$, $\mathrm{a} 3, \mathrm{a} 4)$ and $\mathrm{B}=(\mathrm{b} 1, \mathrm{~b} 2, \mathrm{~b} 3, \mathrm{~b} 4)$, then

$$
\begin{aligned}
& P(A)=\frac{a_{1}+2 a_{2}+2 a_{3}+a_{4}}{6} \\
& P(B)=\frac{b_{1}+2 b_{2}+2 b_{3}+b_{4}}{6}
\end{aligned}
$$

\subsection{Proposed Similarity Measure}

Let $0 \leq \mathrm{x} \leq 1$ and $0 \leq \mathrm{y} \leq 1$. The similarity between $\mathrm{x}$ and $\mathrm{y}$ is defined as:

$$
S(x, y)=1-|\sqrt{x}-\sqrt{y}|
$$

Advantage of using the formula is that small absolute of difference corresponds to a high degree of similarity, whereas two components with a large absolute difference are considered to be very dissimilar.

Now, the degree of similarity between the GTrFN s can be calculated using the formula:

$$
S(A, B)=\left(S\left(h_{A}, h_{B}\right) x \prod_{i=1}^{4} S\left(a_{i}, b_{i}\right)\right)^{\frac{1}{3}}
$$

\section{COMPARATIVE STUDY}

In this section, fifteen sets of generalized trapezoidal fuzzy numbers has been presented. Some of the numbers have been taken from the existing research works and the same has been used to perform the comparative study. Comparison of the calculated results obtained using the proposed similarity measure and the existing similarity measures presented in section 3, has been presented in Table 6.1[11]

\section{1st Set of GTrFN}

$\mathrm{A}=(0.2,0.3,0.5,0.6 ; 1.0) \& \mathrm{~B}=(0.2,0.3,0.5,0.6 ; 1.0)$

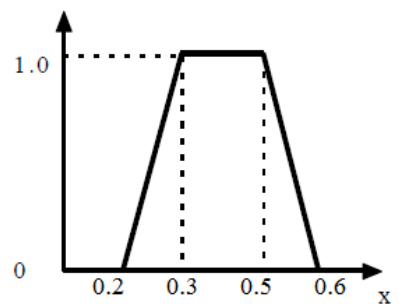

\section{2nd Set of GTrFN:}

$\mathrm{A}=(0.2,0.3,0.5,0.6 ; 1.0) \& \mathrm{~B}=(0.2,0.4,0.4,0.6 ; 1.0)$

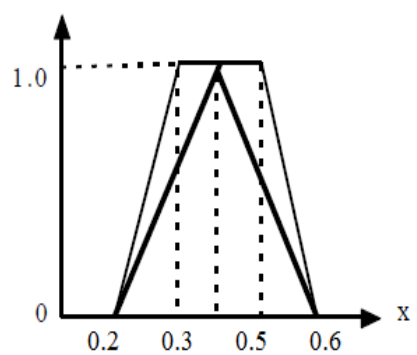

3. 3rd Set of GTrFN:

$\mathrm{A}=(0.2,0.3,0.5,0.6 ; 1.0) \& \mathrm{~B}=(0.6,0.8,0.8,1 ; 1.0)$

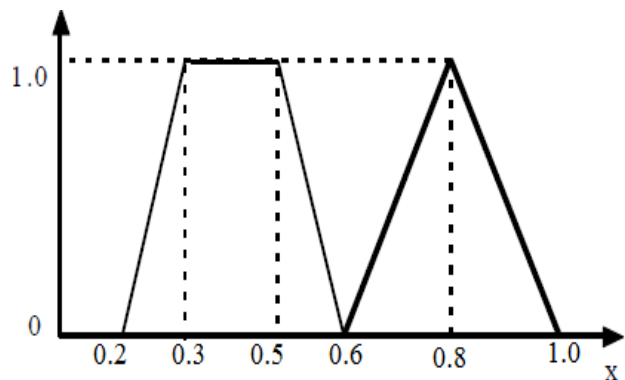

\section{4rth Set of GTrFN:}

$\mathrm{A}=(0.2,0.3,0.5,0.6 ; 1.0) \& \mathrm{~B}=(0.6,0.7,0.9,1 ; 1.0)$

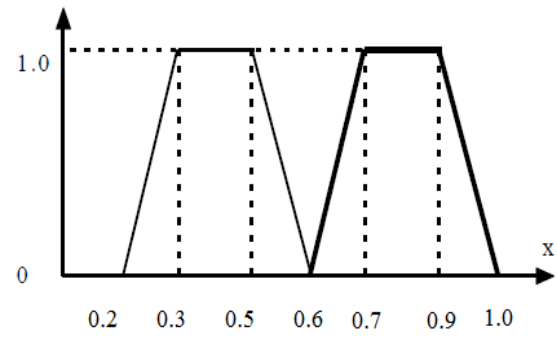

\section{5th Set of GTrFN:}

$\mathrm{A}=(0.2,0.3,0.5,0.6 ; 1.0) \& \mathrm{~B}=(0.2,0.3,0.5,0.6 ; 0.8)$

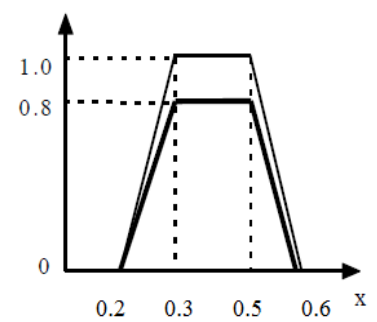

\section{6th Set of GTrFN:}

$\mathrm{A}=(0.5,0.5,0.5,0.5 ; 1.0) \& \mathrm{~B}=(0.5,0.5,0.5,0.5 ; 1.0)$

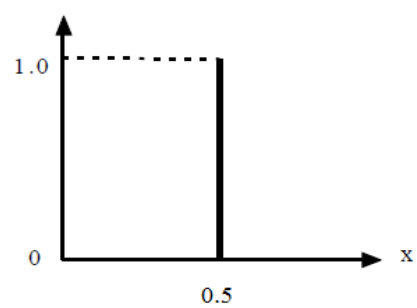


7. 7th Set of GTrFN:

$\mathrm{A}=(0.5,0.5,0.5,0.5 ; 1.0) \& \mathrm{~B}=(0.5,0.5,0.5,0.5 ; 0.8)$

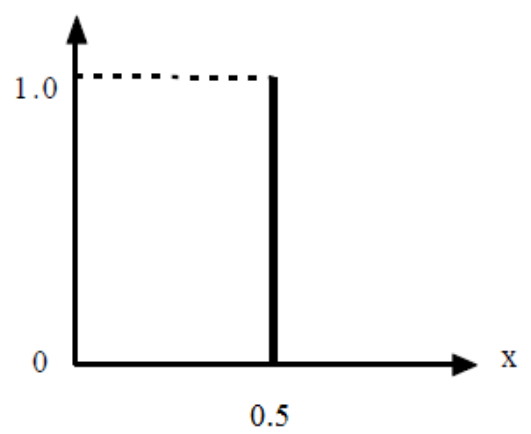

8. 8th Set of GTrFN:

$\mathrm{A}=(0.3,0.3,0.3,0.3 ; 1.0) \& \mathrm{~B}=(0.5,0.5,0.5,0.5 ; 1)$

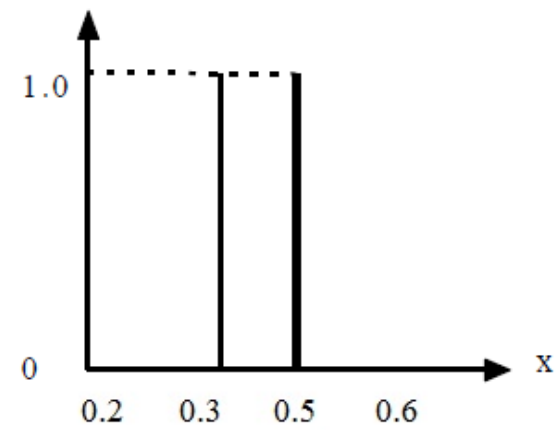

9. 9th Set of GTrFN:

$\mathrm{A}=(0.3,0.3,0.4,0.4 ; 1.0) \& \mathrm{~B}=(0.5,0.5,0.7,0.7 ; 1)$

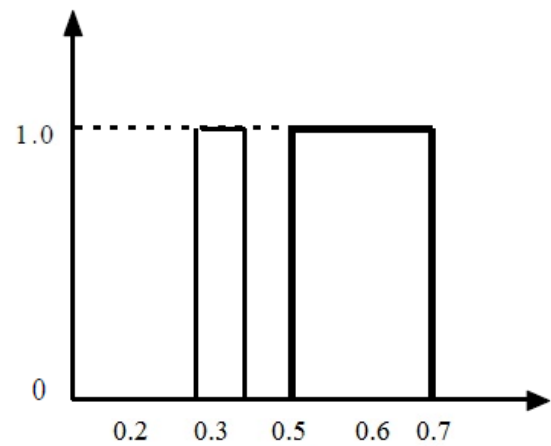

10. 10th Set of GTrFN:

$\mathrm{A}=(0.2,0.2,0.3,0.3 ; 1.0) \& \mathrm{~B}=(0.5,0.5,0.6,0.6 ; 1)$

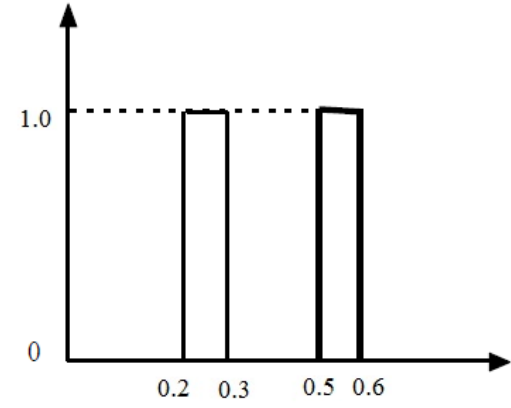

11. 11th Set of GTrFN:

$\mathrm{A}=(0.1,0.2,0.3,0.4 ; 1.0) \& \mathrm{~B}=(0.4,0.5,0.6,0.7 ; 1)$

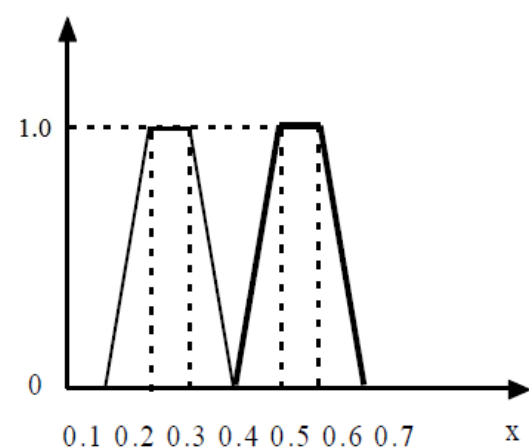

12. 12th Set of GTrFN:

$\mathrm{A}=(0.2,0.3,0.40 .5 ; 1.0) \& \mathrm{~B}=(0.2,0.2,0.2,0.5 ; 1)$

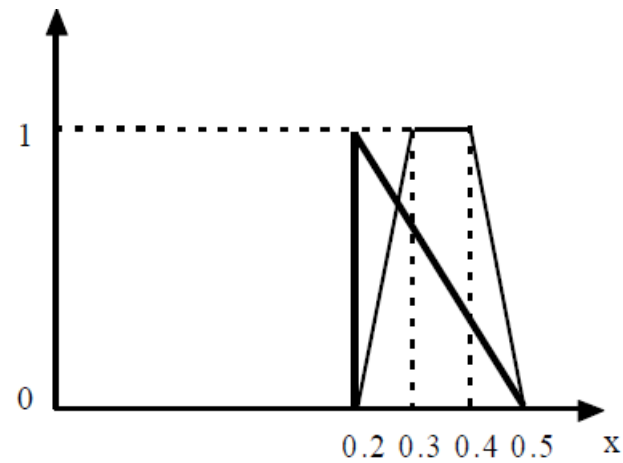

13. 13th Set of GTrFN:

$\mathrm{A}=(0.2,0.3,0.40 .5 ; 1.0) \& \mathrm{~B}=(0.1,0.4,0.4,0.4 ; 1)$

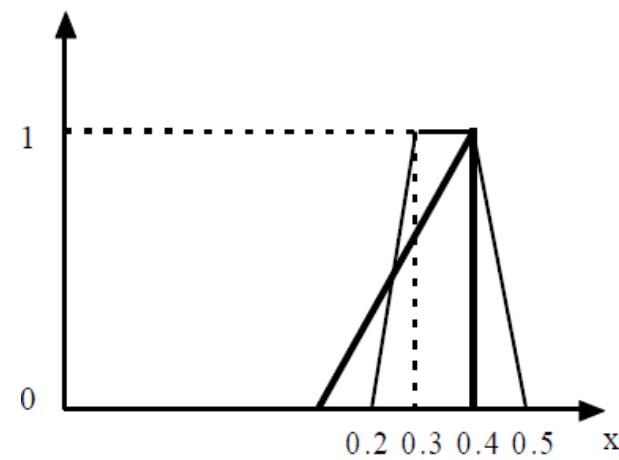

14. 14th Set of GTrFN:

$\mathrm{A}=(0,0,0.30 .3 ; 1.0) \& \mathrm{~B}=(0.2,0.4,0.4,0.5 ; 1)$

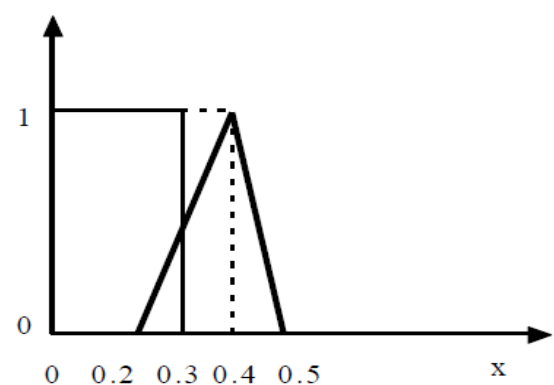




\section{15th Set of GTrFN:}

$\mathrm{A}=(0.2,0.3,0.50 .6 ; 1.0) \& \mathrm{~B}=(0.3,0.4,0.4,0.5 ; 1)$

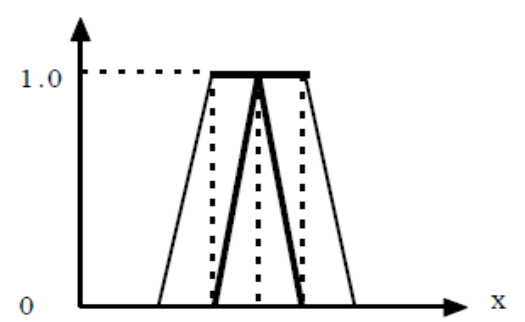

$\begin{array}{lllllllll}0.2 & 0.3 & 0.4 & 0.5 & 0.6\end{array}$

Table 6.1 Comparison of the Calculation Results of the Proposed Similarity Measure with the Existing Methods

\begin{tabular}{|c|c|c|c|}
\hline S. No & $\mathbf{S}_{\mathbf{C}}(\mathbf{A}, \mathbf{B})$ & $\mathbf{S}_{\mathbf{H C}}(\mathbf{A}, \mathbf{B})$ & $\mathbf{S}(\mathbf{A}, \mathbf{B})$ \\
\hline 1. & 1 & 1 & 1 \\
\hline 2. & 0.95 & 1 & 0.947 \\
\hline 3. & 0.6 & 0.715 & 0.652 \\
\hline 4. & 0.6 & 0.715 & 0.655 \\
\hline 5. & 1 & 1 & 0.964 \\
\hline 6. & 1 & 1 & 1 \\
\hline 7. & 1 & 1 & 0.946 \\
\hline 8. & 0.8 & 0.834 & 0.794 \\
\hline 9. & 0.75 & 0.80 & 0.765 \\
\hline 10. & 0.7 & 0.77 & 0.69 \\
\hline 11. & 0.7 & 0.77 & 0.678 \\
\hline 12. & 0.925 & 0.91 & 0.902 \\
\hline 13. & 0.925 & 1 & 0.903 \\
\hline 14. & 0.775 & 0.811 & 0.539 \\
\hline 15. & 0.9 & 1 & 0.893 \\
\hline
\end{tabular}

\section{DISCUSSION}

- Set 1 and 6 presents the similar GTrFNs as calculation of similarity between the fuzzy numbers is found to be 1 .

- For set 3 and 4, using the existing method to calculate the similarity shows that the set presents same fuzzy numbers while calculation using the proposed method is showing the difference between the results obtained i.e., fuzzy numbers are not similar. Hence, proposed method is more accurate in this case because the sets represent different fuzzy numbers. Similar case has been found with set 5 and 6; set 9 and 11 ; and set 12 and 13 .

\section{CONCLUSION}

A novel measure for computing the degree of similarity between two generalized trapezoidal fuzzy numbers has been presented. Comparative study with the existing similarity measure shows the proposed method is accurate and error rate is decreased. It is simple and robust method to calculate the degree of similarity. Using the proposed method ambiguity between different sets of GTrFNs is not obtained.

\section{REFERENCES}

[1] S. J. Chen and S. M. Chen, "Fuzzy Risk Analysis Based on Similarity Measures of Generalized Fuzzy Numbers", IEEE Transactions on Fuzzy Systems, vol. 11, no. 1, (2003), pp. 45-56.

[2] Y. Deng, W. Shi, F. Du and Q. Liu, "A New Similarity Measure of Generalized Fuzzy Numbers and Its Application to Pattern Recognition", Pattern Recognition Letters, vol. 25, no. 8, (2004), pp. 875-883.

[3] C. B. Chen and C. M. Klein, "A Simple Approach to Ranking a Group of Aggregated Fuzzy Utilities", IEEE Transactions on Systems, Man, and Cybernetics - Part B: Cybernetics, vol. 27, no. 1, (1997), pp. 26-35.

[4] S. M. Chen, "A New Approach to Handling Fuzzy Decision-Making Problems", IEEE Transactions on Systems, Man, Cybernetics, vol. 18, no. 6, (1988), pp. 1012-1016.

[5] R. Kangari and L. S. Riggs, "Construction Risk Assessment by Linguistics", IEEE Transactions on Engineering Management, vol. 36, no. 2, (1989), pp. 126-131.

[6] K. J. Schmucker, "Fuzzy Sets, Natural Language Computations, and Risk Analysis", Rockville, MD: Computer Science, (1984).

[7] C. T. Lin and C. T. Chen, "A Fuzzy-Logic-Based Approach for New Product Go/Nogo Decision at the Front End", IEEE Transactions on Systems, Man, Cybernetics-Part A: Systems and Humans, vol. 34, no. 1, (2004), pp. 132-142.

[8] S. M. Chen, "New Methods for Subjective Mental Workload Assessment and Fuzzy Risk Analysis", Cybernetics and Systems, vol. 27, no. 5, (1996), pp. 449472 .

[9] S. M. Chen, "Aggregating Fuzzy Options in the Group Decision-Making Environment", Cybernetics \& Systems, vol. 29, no. 4, (1998), pp. 363-376.

[10] C. H. Hsieh and S. H., "Chen. Similarity of Generalized Fuzzy Numbers with Graded Mean Integration Representation", Proceedings of the 8th International Fuzzy Systems Association World Congress, Taipei, Taiwan, (1999), pp. 551-555.

[11] Yixiang Zhou, "A Novel Similarity Measure for Generalized Trapezoidal Fuzzy Numbers and its Application to Decision-Making”, International Journal of u- and e- Service, Science and Technology Vol.9, No. 3 (2016),pp.131-148 http://dx.doi.org/10.14257/ijunesst. 2016.9.3.14. 